\title{
Perfil de los niveles séricos de eritropoyetina y citocinas proinflamatorias en pacientes con anemia de trastornos crónicos secundaria a infección
}

\author{
F. POVEDA GÓMEZ***, J. CAMACHO SILES, E. QUEVEDO MORALES*, \\ A. FERNÁNDEZ ZAMORANO**, R. CODOCEO ALQUINTA**, F. ARNALICH \\ FERNÁNDEZ*, M. SEMPERE ALCOCER****
}

Servicio de Medicina Interna, *Hematología y **Bioquímica. Hospital Universitario La Paz. Madrid. Áreas de ***Medicina Interna y ****Biotecnología. Hospital Costa del Sol. Marbella. Málaga.

\author{
PATTERN OF ERYTHROPOIETIN AND PROINFLAMMATORY CYTO - \\ KINES IN SERUM FROM PATIENTS WITH ANEMIA OF CHRONIC \\ DISORDERS SECONDARY TO INFECTION
}

\section{RESUMEN}

Objetivo: Evaluar el comportamiento de la eritropoyetina (EPO) y determinadas citocinas proinflamatorias en la anemia de trastornos crónicos (ATC) de origen infeccioso.

Métodos: Determinación secuencial en suero mediante enzimoinmunoanálisis de interleucina-1 beta (IL-1 $\beta$ ), factor de necrosis tumoral alfa (TNF- $\alpha$ ), interferón gamma (IFN- $\gamma$ ), interleucina-6 (IL-6) y eritropoyetina (EPO) en 25 pacientes con enfermedades de origen bacteriano, curso clínico prolongado y criterios de ATC. Se analizó la relación de estos mediadores con la anemia y con el metabolismo del hierro.

Resultados: Los niveles de EPO experimentaron un descenso significativo respecto al nivel inicial, normalizándose en el control tardío $(18,04 \pm 19,10$ vs $8,56 \pm 4,72 \mathrm{UI} / \mathrm{mL} ; \mathrm{p}<0,001$; rango normal: $4-15$ $\mathrm{mUI} / \mathrm{mL}$ ). Inicialmente, existía correlación inversa no significativa entre los niveles de EPO y la concentración de hemoglobina $(\mathrm{r}=-0,115, \mathrm{NS})$, alcanzándose significación estadística en el control tardío $(r=-0,446$; $p$ < $0,05)$. Existía correlación inversa entre el hematocrito y los niveles del TNF- $\alpha(r=0,467 ; p<0,05)$ y entre la hemoglobina y la transformación logarítmica del TNF- $\alpha(r=0,424 ; p<0,001)$. La IL-6 mostró correlación inversa con la hemoglobina $(r=0,366 ; p<0,05)$ y con la sideremia $(r=-$ $0,614 ; \mathrm{p}<0,001)$, mientras que se correlacionó directamente con los niveles de la EPO $(r=0,297 ; \mathrm{p}<0,05)$.

Conclusiones: Una deficiente respuesta de la eritropoyetina y la acción del TNF pueden implicarse en la patogenia de la ATC de origen infeccioso. La correlación positiva entre la IL-6 y la EPO sugiere una implicación proeritropoyética de la primera en respuesta a la anemia.

\begin{abstract}
Objective: To evaluate the pattern of erythropoietin (EPO) and some proinflammatory cytokines in the anemia of chronic disorders $(A C D)$ secondary to infection

Methods: Sequential determination in serum of interleukin-1 beta $(I L-1 \beta)$, necrosis tumoral factor alpha $(T N F-\alpha)$, gamma interferon (IFN$\gamma)$, interleukin-6 (IL-6), and erythropoietin (EPO) in 25 patients with chronic bacterial infectious diseases and ACD criteria. We evaluated the relationship of these mediators with the anemia and the iron metabolism.

Results: Serum EPO levels significatively decreassed compared with initial values, and the last control was in normal rank (18.04 $\pm 19.10 \mathrm{vs}$. $8.56 \pm 4.72 \mathrm{UI} / \mathrm{mL} ; p<0.001$; normal rank: $4-15 \mathrm{mUI} / \mathrm{mL})$. In the first control, there was a negative and non significative correlation between the EPO levels and the hemoglobin concentration $(r=-0.115, N S)$, rea ching significance in the last control $(r=-0.446 ; p<0.05)$. There was negative correlation between the hematocrit and TNF- $\alpha$ levels $(r=$ $0.467 ; p<0.05)$ and between the haemoglobin values and the log of serum TNF- $\alpha(r=0.424 ; p<0.001)$. An inverse correlation between the IL-6 levels and both, the hemoglobin concentration and the serum iron was found, and there was a direct correlation between this cytokine values and the EPO levels.

Conclussions: Blunted response of erythopoietin and the action of $T N F$ may contribute to the pathogenesis of ACD secondary to infection. Positive correlation between $I L-6$ and EPO suggest a proerythropoietic action of $I L-6$ in response to the anemia.
\end{abstract}

KEY WORDS: Anemia. Infection. Erythropoietin. Cytokines.

PALABRAS CLAVE: Anemia. Infección. Eritropoyetina. Citocinas.

Póveda Gómez F, Camacho Siles J, Quevedo García E. Fernández Zamorano A, Cocodeo R, Arnalich Fernández F, Sempere Alonso M. Perfil de los niveles séricos de eritropoyetina y citocinas proinflamatorias en pacientes con anemia de trastornos crónicos secundaria a infección. An Med Interna (Madrid) 2001; 18: 298-304.

\section{INTRODUCCIÓN}

Los verdaderos mecanismos patogénicos implicados en el desarrollo de la anemia de trastornos crónicos (ATC) continúan actualmente siendo inciertos, si bien se piensa que ésta pudiera tener un origen multifactorial, donde podrían concurrir diversos trastornos que incluirían: una moderada reducción de la super- vivencia eritrocitaria (1), un secuestro del hierro por parte de las células del sistema mononuclear-fagocítico con el consecuente déficit de aporte del mismo a los precursores eritroides $(2,3)$ y una inadecuada respuesta del órgano hematopoyético ante la anemia $(4,5)$. Respecto al papel preciso que puedan desempeñar en estos procesos la eritropoyetina (EPO) y, por otro lado, determinados mediadores de la respuesta inflamatoria, como las citocinas, no está perfectamente establecido.

Trabajo aceptado: 12 de Enero de 2001

Correspondencia: Francisco Poveda Gómez. Unidad de Medicina Interna. Hospital “Costa del Sol”. Crta. Nacional 340, km 187. 29600-Marbella. Málaga. 
En los últimos años, algunos autores, apoyados en técnicas de cultivo in vitro de precursores eritroides, han tratado de implicar a algunos mediadores celulares (linfocitos $\mathrm{T}$ ) y/o humorales (IL-1, IL-6, TNF e INF-gamma) de la inflamación en el fracaso regenerativo medular característico de la ATC $(6,7,8)$. Por otra parte, dado que está bien establecido que en esta entidad la inhibición de la eritropoyesis se correlaciona con la severidad de la anemia (9) y con la actividad inflamatoria $(10,11)$, es concebible que estas citocinas pudieran tener un papel en la inhibición de la respuesta eritropoyética. Igualmente, en este tipo de pacientes se han encontrado concentraciones séricas elevadas de ciertas citocinas proinflamatorias (IL-1, TNF), fundamentalmente cuando se trata de procesos febriles (12) e infecciosos (13). Constituyendo éstas las principales líneas de investigación abiertas actualmente en el estudio de la ATC, nos propusimos evaluar de forma simultánea en pacientes con enfermedades infecciosas bacterianas de curso prolongado y criterios de ATC la respuesta de la eritropoyeina (EPO) y la eventual influencia que sobre la eritropoyesis pudieran ejercer citocinas moduladoras de la respuesta inflamatoria.

\section{PACIENTES Y MÉTODOS}

Pacientes. Se estudió un grupo de 25 pacientes ingresados en el Departamento de Medicina con enfermedad febril de etiología infecciosa probable o definida de al menos 3 semanas de evolución, con manifestaciones de síndrome de respuesta inflamatoria sistémica (14) y con presencia de anemia de trastornos crónicos (ATC), según los criterios diagnósticos de Lee y cols. (15). Se descartó la coexistencia de déficit tisular férrico excluyendo aquellos pacientes con una feritina sérica inferior a $125 \mu \mathrm{g} / \mathrm{L}$; según establecieron Witte y cols.(16), la probabilidad de déficit tisular férrico es menor del $6 \%$ con una ferritinemia superior a $100 \mu \mathrm{g} / \mathrm{L}$. Fueron excluidos pacientes con cualquiera de las siguientes condiciones: edad superior a 80 o inferior a 18 años; hipoxemia moderada-severa $(\mathrm{PO} 2<60 \mathrm{mmHg}$ y/o Sat.O2 < 90\%); insuficiencia renal (creatinina sérica $>1,5 \mathrm{mg} / \mathrm{dL}$ ); endocrinopatía subyacente (hipotiroidismo, hiperparatiroidismo e hipopituitarismo); hepatopatía moderada-severa; alcoholismo crónico; terapéutica en los últimos 3 meses con hierro, vitaminas o fármacos potencialmente mielosupresores; tratamiento concomitante o durante el mes previo al ingreso con corticoides; neoplasia subyacente; artritis reumatoide y otras enfermedades colágeno-vasculares; e inmunodeficiencia primaria conocida o infección por V.I.H.

En la medida que el estudio a realizar en cada paciente era secuencial, con evaluaciones a lo largo de todo el proceso infeccioso, cada paciente se constituía en su propio control. De esta forma, se consideraban como controles de las diferentes variables incluidas en el protocolo los valores obtenidos al final del seguimiento, coincidiendo con curación de los respectivos procesos infecciosos (al menos 90 días después de la evaluación inicial). Así mismo, tanto para la EPO como para las citocinas se consideraron como valores de referencia los resultados obtenidos previamente por el mismo laboratorio, y en similares condiciones técnicas, en una población adulta sana.

Determinaciones de laboratorio. De las citocinas plasmáticas estudiadas, IL- $1 \beta$, TNF- $\alpha$, IFN- $\gamma$ e IL-6, se determinaron por enzimoinmunoanálisis (Medgenix ${ }^{\mathrm{TM}}$. Boston) en placa microtiter recubierta de anticuerpo monoclonal marcado con peroxidasa de caballo. Es un sistema oligloconal en el que los diferentes anticuerpos monoclonales se unen a diferentes epítopes de las citocinas determinadas con objeto de aumentar la sensibilidad y especificidad del método y acortar el tiempo de incubación. El ensayo se realizó sobre plasma sin ningún tratamiento o extracción. La eritropoyetina (EPO) se determinó en suero mediante enzimoinmunoanálisis (bioMèrieux ${ }^{\mathrm{TM}}$. Lyon) en placa microtiter recubierta de anticuerpo monoclonal anti-EPO marcada con peroxidasa de rábano blanco silvestre. La determinación se efectúa según una técnica de sandwich que utiliza dos anticuerpos monoclonales dirigidos frente a diferentes epítopes de la eritropoyetina. Las determinaciones bioquímicas rutinarias se realizaron en un autoanalizador Hitachi 737. Las determinaciones hematológicas mediante un autoanalizador Technicon H1 system. Los niveles en suero de la ferritina y de la transferrina se cuantificaron mediante radioinmunoanálisis.

Análisis estadístico. El tratamiento estadístico de los datos se llevó a cabo mediante el programa SPSS/PC. Los resultados finales de las variables cuantitativas se expresaron como media más/menos desviación estándar $(\mathrm{X} \pm \mathrm{SD})$. En el caso de las citocinas se calculó también el error estándar de la media (SEM), el cual aparece reflejado en los diferentes histogramas. La comparación de las medias se realizó mediante la utilización de la $t$ de Student y los test no paramétricos de Wilcoxon (datos apareados) y de la $U$ de Mann - Whitney (muestras independientes). Para estudio de la asociación entre variables se calculó el coeficiente de correlación de Pearson con un análisis de regresión simple. En todas las pruebas de hipótesis se consideró con nivel de significación $\mathrm{p}$ $<0,05$.

\section{RESULTADOS}

Características clínicas y respuesta de la eritropoyetina ante la anemia. Las principales características clínicas y hematológicas de los pacientes con ATC aparecen recogidas en la Tabla I. En el seguimiento evolutivo de los pacientes se observó una normalización de la concentración de hemoglobina y del hematocrito a la curación de los diversos procesos infecciosos, observándose diferencia significativa entre los valores medios de estos parámetros hematológicos al comparar los resultados obtenidos al ingreso con los del final del seguimiento de los pacientes (hemoglobina: 10,4 $\pm 1,1$; SEM 0,$2 ;$ vs $14,3 \pm 0,3 \mathrm{~g} / \mathrm{dL}$; SEM 0,3; hematocrito: $31,7 \pm 3,1$; SEM 0,6; vs 40,6 $\pm 4,5 \%$; SEM 0,9; $p<0,05$ en ambas comparaciones). Igualmente, la concentración media de eritropoyetina experimentó un significativo descenso respecto al nivel inicial, situándose en el control tardío dentro de los valores de normalidad de nuestro laboratorio $(18,04 \pm 19,10$; SEM 3,89 ; vs. 8,56 \pm 4,72 UI/mL; SEM 0,94; $\mathrm{p}<0,001$; rango normal: 4-15 mUI/mL) (Fig. 1).

Comportamiento de las citocinas proinflamatorias. Los pacientes presentaron en el control inicial unas concentraciones de las cuatro citocinas evaluadas superiores al rango de normalidad obtenido en adultos sanos, y significativamente mayores a los obtenidos en el control tardío a la curación de los procesos en el caso del TNF- $\alpha$ y la IL-6 (IL-1 $\beta$ : $10,45 \pm$ 2,92 vs. $5,37 \pm 0,93 \mathrm{pg} / \mathrm{mL}, \mathrm{p} 0,070$; TNF- $\alpha$ : 31,97 $\pm 4,53$ vs. 


\section{TABLA I}

CARACTERÍSTICAS CLÍNICAS DE LA PO BLACIÓ N ESTUDIADA

\begin{tabular}{|c|c|}
\hline Número (n) & 25 \\
\hline Edad (años; media \pm EEM y ranto) & $54 \pm 18 ; 23-78$ \\
\hline $\operatorname{Sexo}(H / M)$ & $15 / 10$ \\
\hline \multicolumn{2}{|l|}{ Diagnóstico clínico } \\
\hline Tuberculosis pleuropulmonar & 11 \\
\hline Tuberculosis pericárdica & 1 \\
\hline Tuberculosis ósea & 1 \\
\hline Neumonía necrotizan & 5 \\
\hline Empiema pleural & 4 \\
\hline Abceso renal & 2 \\
\hline Endocarditis subaguda & 1 \\
\hline \multicolumn{2}{|l|}{ Diagnóstico microbiológico } \\
\hline M ycobacterium tuberculosis & 13 \\
\hline Streptococcus pneumoniae & 1 \\
\hline Klebsiella penumoniae & 1 \\
\hline Staphilococcus aureus & 1 \\
\hline Eschericia coli & 1 \\
\hline Enterobacter aerogenes & 1 \\
\hline Streptococus viridans & 1 \\
\hline Serratia marcesens & 1 \\
\hline Enterobacter cloacae & 1 \\
\hline Acynetobacter calcoacéticus & 1 \\
\hline Bacxteroides fragilis & 1 \\
\hline Xantomona maltophila & 2 \\
\hline Polimicrobiano & 5 \\
\hline
\end{tabular}

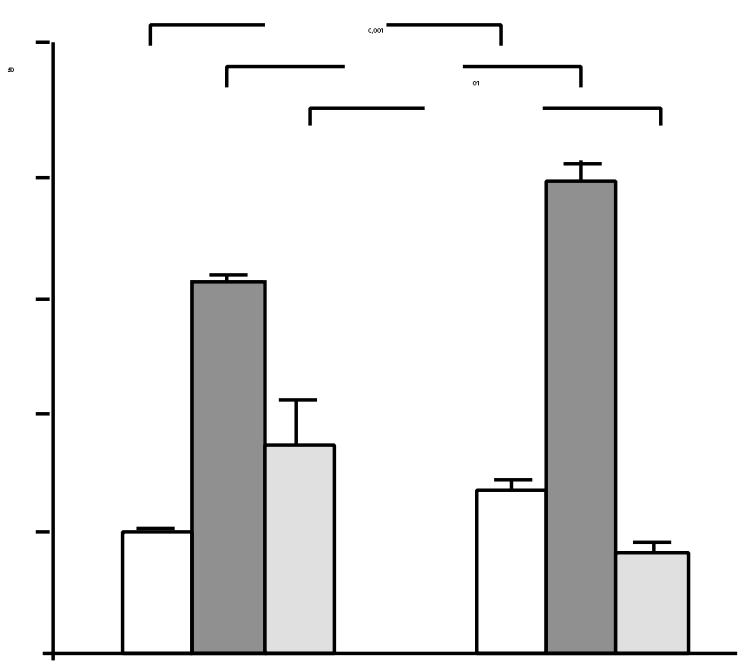

Fig. 1. Concentración de hemoglobina, hematocrito y niveles séricos de EPO (M edia \pm EEM ) al ingreso y a la curación de los procesos infecciosos con ATC $(n=25)$.

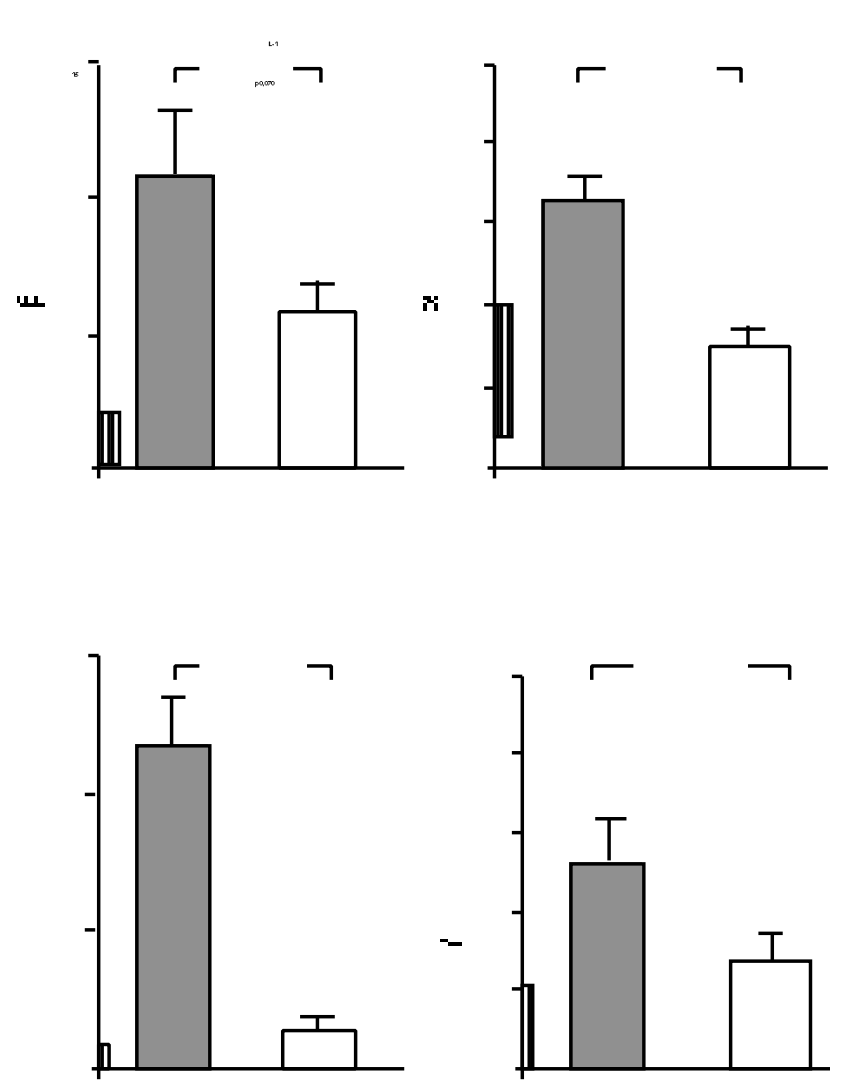

Fig. 2. Niveles plasmáticos de IL-1 $\beta$, TNF- $\alpha$, I L- 6 e IFN- $\gamma$ (media \pm EEM) al ingreso y a la curación de los procesos infecciosos en pacientes con ATC $(n=25)$. Sobre el eje vertical se referencia el rango normal en 80 adultos sanos (IC 95\%).

$14,83 \pm 2,26$ pg/mL, p 0,003; IL-6: $116 \pm 19,26$ vs. $13,65 \pm$ $4,91 \mathrm{pg} / \mathrm{mL}, \mathrm{p} 0,001 ;$ IFN- $\gamma: 0,512 \pm 0,120$ vs $0,268 \pm 0,057$ $\mathrm{UI} / \mathrm{mL}, \mathrm{p}$ 0,093) (Fig. 2).

Relación de la EPO y citocinas proinflamatorias con los marcadores de la anemia. Al evaluar la relación entre la respuesta de la eritropoyetina y la anemia se observaron en los pacientes con ATC correlaciones inversas no significativas entre los niveles séricos de EPO y los dos principales indicadores de la anemia, cuales son la concentración de hemoglobina $(r=-0,115, \mathrm{p} 0,594)$ y el hematocrito $(r=-0,046, \mathrm{p} 0,830)$. Sin embargo, dicha correlación inversa entre la hemoglobina y los niveles de EPO alcanzaba significación estadística en el control analítico tardío a la curación de los diversos procesos infecciosos y, por lo tanto, una vez resuelta la anemia $(\mathrm{r}=-$ 0,0446; p 0,020) (Fig. 3a). Respecto a las citocinas proinflamatorias, se encontró que en el control inicial existía correlación inversa estadísticamente significativa entre el hematocrito y los niveles del TNF- $\alpha(r=0,467 ; p=0,010)$ (Fig. 3a). Respecto a la concentración de hemoglobina, mostró un comportamiento parecido, si bien en este caso con la transformación logarítmica del TNF- $\alpha(\mathrm{r}=0,424 ; \mathrm{p}=0,002)$ (Fig. 3b). No se observaron en los pacientes con ATC otro tipo de correlaciones similares en el caso de la IL- $1 \beta$ y del IFN- $\gamma$. (hemoglobina-IL-1 $\beta$ : $r=0,030$, p 0,990; hematocritoIL-1 $\beta: r=0,148$, p 0,172; hemoglobina-IFN- $\gamma: r=-0,179$, 

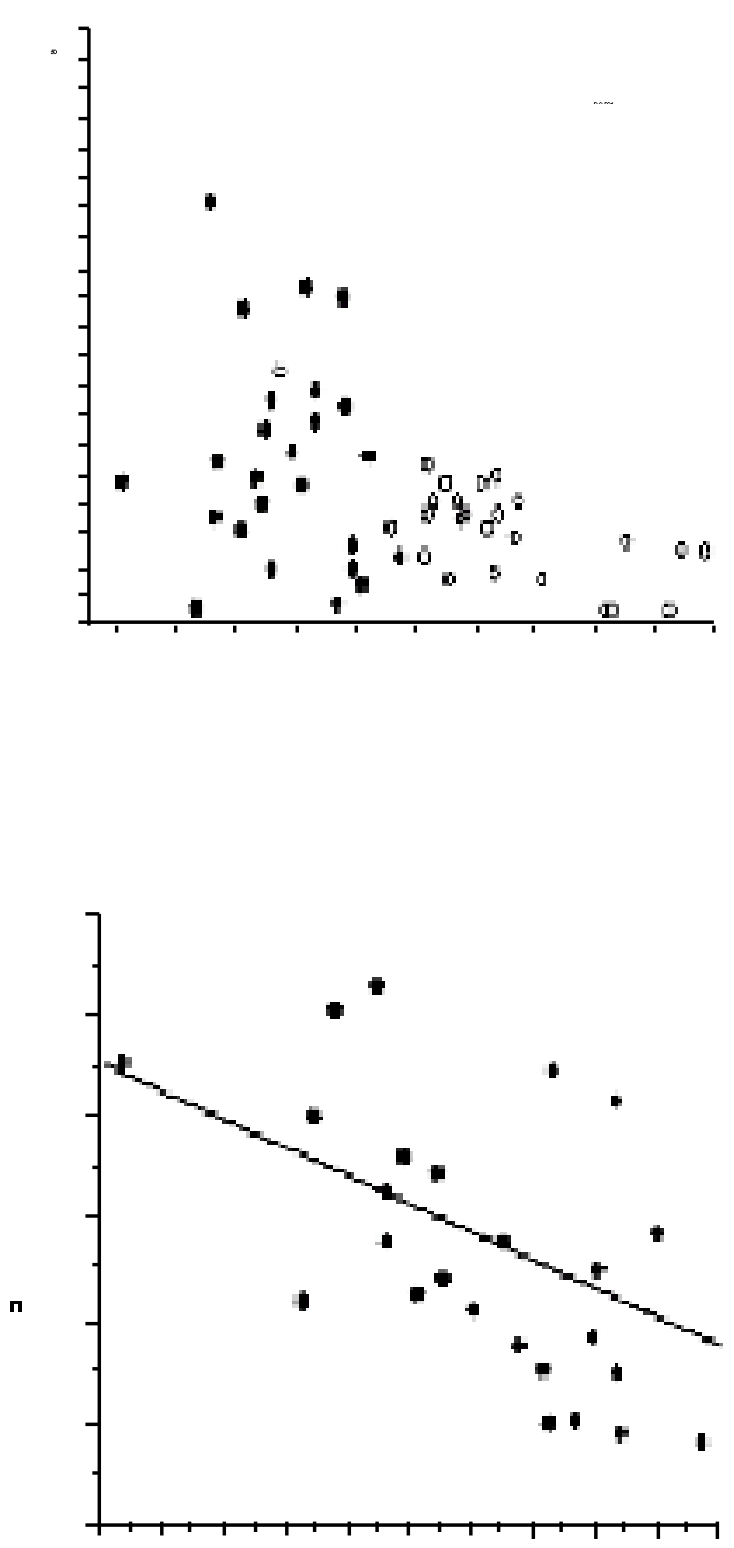

Fig. 3 a) Análisis de regresión entre la concentración de hemoblogina y los niveles séricos de EPO en los pacientes con ATC, representándose las determinaciones al ingreso $(\bullet)$ y a la curación de los procesos infeccciosos y resolución de la anemia (o). b) Análisis de regresión entre la concentración de hemoglobina decimal de los niveles plasmáticos del TNF- $\alpha$ en los pacientes con ATC.

p 0,717; hematocrito-IFN- $\gamma: \mathrm{r}=-0,134, \mathrm{p} 0,553)$. En el caso de la IL-6, se observó una correlación inversa entre la concentración de hemoglobina y los niveles plasmáticos de la IL-6 (r $=0,366 ;$ p 0,021) (Fig. 4a). El estudio de relación entre la eritropoyetina y las diferentes citocinas proinflamatorias evaluadas, mostró que existía una débil correlación directa estadísticamente significativa entre los niveles iniciales de la EPO y la IL-6 ( $r=0.297 ; p=0,047)$ (Fig. 4b).

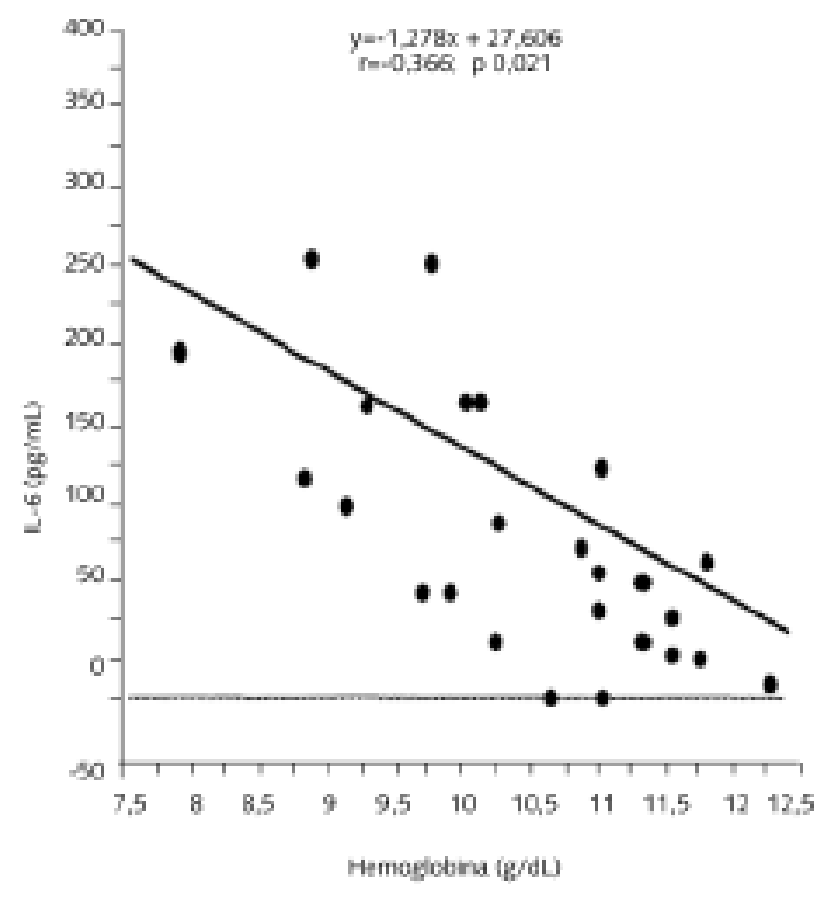

(a)

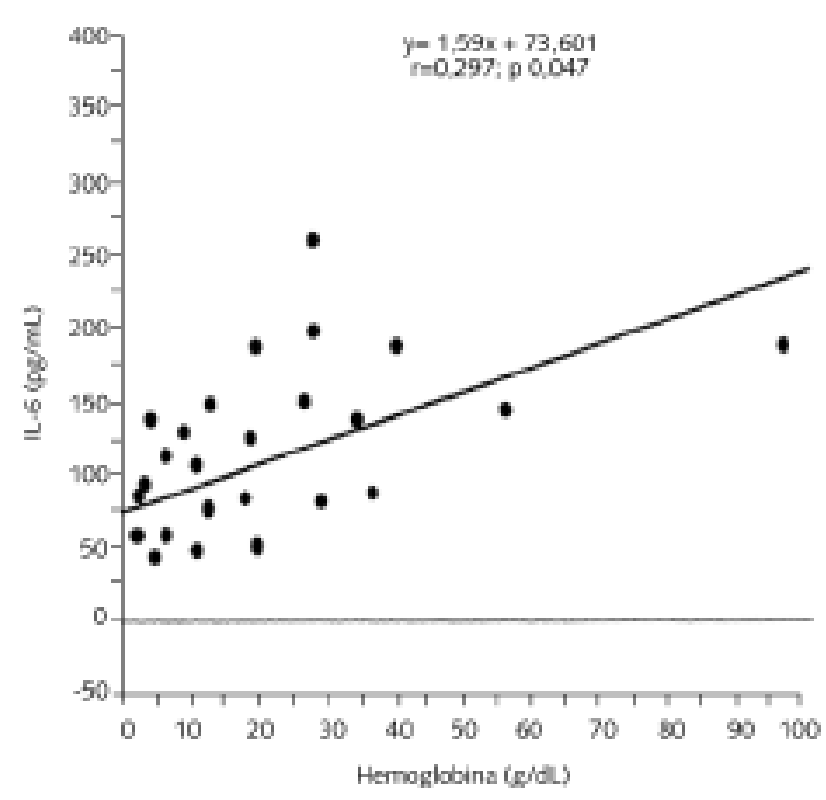

b)

Fig. 4. Análisis de regresión de los niveles plasmáticos de IL- 6 con la concentración de hemoglobina (a) y con los niveles séricos de EPO (a) en los pacientes con ATC.

Relaciones con el metabolismo del hierro. Se evaluó la relación de los marcadores biológicos de la respuesta de fase aguda y de las diversas citocinas estudiadas con una de las alteraciones habitualmente observadas en la anemia de los trastornos crónicos, cual es el trastorno metabolismo del hierro. Se observó una correlación entre la hiposideremia y dos de los principales marcadores de la RFA, concretamente el recuento leucocitario $(r=-0,499, \mathrm{p} 0,001)$ y la VSG $(r=-0,395, \mathrm{p} 0,049)$. Al 
TABLA II

PARÁM ETRO S HEM ATO LÓ GICOS, DEL M ETABO LISM O DEL HIERRO Y DE LA RESPUESTA DE FASE AGUDA

\begin{tabular}{|c|c|c|c|}
\hline Parámetro & Día 1 & Curación & $P$ \\
\hline Eritrocitos $\left(\times 10^{6} \mu \mathrm{L}^{-1}\right)$ & $3,8 \pm 0,4$ & $4,9 \pm 0,3$ & $<0,001$ \\
\hline Hemoglobina $\left(\mathrm{g} \mathrm{dL}^{-1}\right)$ & $10,4 \pm 1,1$ & $14,3 \pm 0,3$ & $<0,001$ \\
\hline Hematocrito $(\%)$ & $31,7 \pm 3,1$ & $40,6 \pm 4,5$ & $<0,001$ \\
\hline VCM (fL) & $82,3 \pm 7,1$ & $84,2 \pm 8,2$ & NS \\
\hline $\mathrm{HCM}(p g)$ & $27,5 \pm 2,7$ & $28,5 \pm 3,1$ & NS \\
\hline $\mathrm{CHCM}\left(g \mathrm{dL}^{-1}\right)$ & $33,0 \pm 0,3$ & $33,2 \pm 0,4$ & NS \\
\hline Hierro sérico (mg dL ${ }^{-1}$ ) & $36,9 \pm 20,5$ & $103,5 \pm 37,3$ & $<0,001$ \\
\hline Transferrina sérica $\left(\mathrm{mg} \mathrm{dL}^{-1}\right)$ & $266,3 \pm 58,0$ & $240,3 \pm 46,2$ & NS \\
\hline Ferritina sérica $\left(\mathrm{mg} \mathrm{L}^{-1}\right)$ & $443,3 \pm 51,4$ & $142,4 \pm 112,2$ & $<0,05$ \\
\hline Saturación de transferrina (\%) & $7,6 \pm 7,7$ & $37,4 \pm 10,2$ & $<0,05$ \\
\hline Temperatura axilar $\left({ }^{\circ} \mathrm{C}\right)$ & $38,5 \pm 0,9$ & $36,3 \pm 0,3$ & $<0,05$ \\
\hline Leucocitos $\left(\mu^{-1}\right)$ & $9,064 \pm 3,257$ & $5,755 \pm 1,645$ & $<0,05$ \\
\hline VSG (mm/1ah) & $104,3 \pm 26,1$ & $37,8 \pm 13,5$ & $<0,001$ \\
\hline Haptoglobina (mg dL $\mathrm{dL}^{-1}$ ) & $263,3 \pm 138,6$ & $135,0 \pm 76,2$ & $<0,001$ \\
\hline
\end{tabular}

Valores expresados como media \pm EEM . NS = diferencia no signiticativa.

evaluar el comportamiento de las citocinas respecto a este trastorno del metabolismo del hierro, se observó como los niveles plasmáticos de la IL-6 guardaban correlación inversa con la sideremia $(r=-0,614, p 0,001)$. Otro tipo de citocinas proinflamatorias no mostraron correlación con la hiposideremia, ni con otros parámetros del metabolismo del hierro.

\section{DISCUSIÓN}

Los pacientes incluidos en nuestro estudio, con infecciones de curso prolongado, presentaron unos índices corpusculares y unos parámetros del metabolismo del hierro acordes con los criterios habituales de ATC. El primero de los aspectos destacables de los resultados de nuestro trabajo en cuanto a probables factores relacionados con este trastorno hematopoyético fue un aparente fracaso en la respuesta del principal factor hematopoyético de la serie roja, la eritropoyetina (EPO), en los pacientes con ATC. Si bien los pacientes con anemia presentaron unos niveles séricos de EPO superiores al rango de normalidad de nuestro laboratorio, no encontramos una correlación significativa entre los principales indicadores de la anemia (concentración de hemoglobina y el hematocrito) y la concentración de eritropoyetina. Sin embargo, esta correlación se recuperaba en el control tardío a la resolución de la enfermedad. A diferencia del grupo crónico, en los pacientes con infecciones agudas, existía una correlación inversa significativa entre el logaritmo de los niveles séricos de EPO y la concentración de hemoglobina. Realmente, esta relación inversa entre el nivel sérico de eritropoyetina y la concentración de hemoglobina suele ser un hecho biológico dependiente del grado de hipoxia tisular, estando presente en condiciones de salud y en cualquier tipo de anemia diferente a la observada en la insuficiencia renal crónica (17). Ha existido gran controversia en los últimos años acerca de la adecuación entre la secreción de la eritropoyetina y el grado de anemia en pacientes con ATC. La mayoría de las investigaciones al respecto se refieren al pro- totipo de ATC, cual es la anemia asociada a la artritis reumatoide (AR). Mientras que algunos investigadores han encontrado en esta entidad unos niveles séricos de EPO apropiadamente elevados al grado de anemia $(18,19)$, Hochberg y cols. (20) y Baer y cols. (21) comunicaron en los pacientes con anemia y AR una menor respuesta en la secreción de EPO que la observada en otros tipos de anemia. Estos últimos hallazgos han sido posteriormente confirmados por otros autores, los cuales demostraron similar respuesta de la EPO en pacientes anémicos con cáncer (22). Respecto al comportamiento de la secreción de EPO en la anemia de las infecciones crónicas, nuestros resultados concuerdan con los obtenidos por Spivack y cols. (23) en pacientes con síndrome de inmunodeficiencia adquirida (SIDA) y por Ebrahim y cols. (24) en pacientes con tuberculosis. Resultados de investigaciones previas de nuestro grupo mostraron la existencia de una deficiente secreción de eritropoyetina en pacientes con ATC, siendo esta falta de respuesta significativamente más manifiesta en el subgrupo etiológico infeccioso que en los otros dos, inflamatorio crónico y neoplásico (25). Posteriormente, hemos comunicado una deficiente secreción de eritropoyetina en pacientes con infección avanzada por el virus de la inmunodeficiencia humana (VIH) (26). Por lo tanto, los resultados del presente trabajo y los procedentes de investigaciones previas sugieren que una inadecuada producción de la EPO ante la anemia constituye uno de los mecanismos implicados en la patogenia de la ATC. Futuras investigaciones deberían ir orientadas a evaluar la eficacia del tratamiento sustitutivo con EPO humana recombinante (rHuEPO) en la anemia de las enfermedades infecciosas crónicas, de la misma forma que se ha experimentado con éxito en la anemia de la AR $(27,28)$.

Otro aspecto destacable fueron los resultados obtenidos al estudiar la relación del TNF- $\alpha$ con los principales indicadores de la anemia. En los pacientes con ATC, los niveles plasmáticos de TNF- $\alpha$ mostraron una correlación inversa significativa con el hematocrito, mientras que la concentración de hemoglobina guardaba una correlación similar con 
el logaritmo decimal del TNF- $\alpha$. Estudios experimentales previos ya han puesto de manifiesto un probable papel de ésta y otras citocinas proinflamatorias en la patogenia de la ATC. En primer lugar, la administración crónica e intermitente de TNF es capaz de provocar anemia en animales de experimentación (29). En segundo lugar, la administración de TNF recombinante con fines terapéuticos a enfermos con cáncer metastásico provocó anemia a todos aquellos pacientes que completaron dicho ensayo clínico (30). Sin embargo, hay poca información sobre los niveles circulantes de esta citocina en pacientes con ATC. Greedyke y cols. (31), evaluaron los niveles plasmáticos de diversas citocinas (IL3, G-CSF, GM-CSF, IL-1 y TNF- $\alpha$ ) en pacientes con ATC, no encontrando diferencias significativas respecto a los controles. Recientemente, Voulgari y cols. (8) han comunicado una elevación significativa de los niveles séricos de TNF- $\alpha$, IL-1 $\beta$ e IL- 6 en pacientes con ATC secundaria a AR. Nuestros resultados, sugieren que el TNF- $\alpha$, una de las principales citocinas implicadas en el proceso inflamatorio, podría comportarse como uno de los factores iniciadores y/o mediadores de la anemia en los procesos inflamatorios crónicos. Los mecanismos por los que ejercería esta mediación son desconocidos, si bien hay evidencias previas que apuntan a que esta acción la ejercería mediante una modulación directa de la eritropoyesis. En este sentido, mediante estudios in vitro, Roodman y cols. (32) y Akahane y cols. (33) demostraron que el TNF inhibía selectivamente la capacidad proliferativa de los principales precursores eritroides, BFU-E y CFU-E. Algunos autores han postulado un segundo mecanismo por el que se implicaría esta citocina en la respuesta eritropoyética, al demostrar la capacidad in vitro del TNF- $\alpha$ para frenar la producción de EPO por determinadas líneas hepatocelulares humanas (HepG2) (34). Sin embargo, en nuestros resultados no hemos encontrado relación alguna entre los niveles circulantes de EPO y los del TNF- $\alpha$ en los pacientes con anemia, lo cual no apoya la posibilidad de que esta última citocina pudiera estar implicada en la falta de respuesta secretora de la EPO ante la anemia, como han apuntado otros autores. Por lo tanto, los papeles de ambas citocinas en la patogenia de la ATC parecen independientes.

Otra citocina proinflamatoria que mostró cierta relación con los indicadores de la anemia fue la IL-6. Otros autores han apuntado previamente la posibilidad de que la IL-6 sea un mediador de la ATC, fundamentalmente en la anemia de la $\operatorname{AR}(8,35)$. Asano y cols. (36) demostraron que la administración a primates de IL-6 recombinante generaba anemia en los mismos. Vreugdenhil y cols. (37) encontraron que los niveles plasmáticos de IL-6 en pacientes con AR eran mayores en aquéllos que presentaban anemia respecto a los pacientes no anémicos, y que estos niveles se correlacionaban con los marcadores de actividad inflamatoria. Un factor que apoyaría esta relación de la IL-6 con la anemia ha sido la correlación inversa que hemos encontrado en los pacientes con anemia entre los niveles plasmáticos de IL-6 y la sideremia, al mismo tiempo que ésta última se correlacionaba también de forma inversa con determinados parámetros de la RFA, como la VSG y el recuento leucocitario. Estos hallazgos sugieren una implicación de la IL-6 en uno de los mecanismos fisiopatológicos tradicionalmente implicado en la
ATC, como es la hiposideremia resultante de un secuestro férrico a cargo del sistema mononuclear fagocítico, en relación directa a la propia respuesta inflamatoria. Por último, al estudiar la relación entre la anemia y la IL-6 encontramos una correlación inversa entre la concentración de hemoglobina y los niveles plasmáticos de la IL-6 en el total de los pacientes. Adicionalmente, los niveles plasmáticos de la IL6 mostraron una correlación directa con la concentración sérica del principal factor eritropoyético, la eritropoyetina. Estos resultados podrían justificar un papel a la IL-6 como factor estimulante de la respuesta eritropoyética, de tal forma que su elevación en los pacientes con anemia constituiría un mecanismo de compensación a la misma y/o simplemente un marcador de la respuesta inflamatoria. Esta hipótesis está sostenida por los resultados complementarios obtenidos en el anteriormente referido trabajo de Vreugdenhil y cols. (37). Estos autores comunicaron como la adición de IL-6 a cultivos celulares de precursores eritroides no inhibía la formación in vitro de colonias. De hecho, la adición a estos cultivos de anticuerpos anti-IL-6 empeoraba la eritropoyesis in vitro, sugiriendo que el efecto de la IL-6 es más estimulador que inhibidor de la eritropoyesis. Por lo tanto, a la vista de nuestros resultados, la IL-6 se perfilaría como una citocina con un papel no claramente definido en la patogenia de la ATC. Al margen de una probable implicación en el trastorno del metabolismo del hierro, parece que constituiría fundamentalmente un factor relacionado con los mecanismos de compensación ante la anemia. Futuras investigaciones que evaluaran la respuesta terapéutica a la IL-6 recombinante en pacientes con ATC podrían aportar más luz acerca del papel real de esta citocina en la patogenia de esta entidad.

Finalmente, han sido apuntados otros mecanismos por los que éstas y otras citocinas proinflamatorias pueden participar en la patogenia de la ATC, influyendo en la respuesta medular a los factores eritropoyéticos o modulando la propia acción de la eritropoyetina. Sin embargo, a la vista de los resultados de nuestro trabajo y de los de otros autores, éstas son cuestiones aún sin resolver. El esclarecimiento de los verdaderos mecanismos biológicos que median esta entidad contribuiría notablemente al establecimiento de estrategias terapéuticas eficaces, y por lo tanto, debe constituir un estímulo para el desarrollo de futuras líneas de investigación en este sentido.

En conclusión, a la vista de nuestros resultados se puede interpretar que una deficiente respuesta de la eritropoyetina puede ser uno de los mecanismos implicados en la patogenia de la anemia de trastornos crónicos asociada a enfermedades infecciosas de curso prolongado. en la patogenia de esta entidad. La correlación inversa encontrada en los pacientes con anemia de trastornos crónicos entre los niveles del TNF$\alpha$ y los principales indicadores hematimétricos de la anemia sugieren que esta citocina proinflamatoria puede comportarse como uno de los factores iniciadores o mediadores de la anemia. Esta implicación es independiente del papel que pueda jugar la eritropoyetina. Aunque el potencial papel de la Interleucina- 6 en el desarrollo de anemia no queda claramente definido, la correlación positiva encontrada entre la IL-6 y la EPO sugiere una implicación proeritropoyética de ésta última en respuesta a la anemia. 


\section{Bibliografía}

1. Witte DL, Kraemer DF, Johnson GF, Dick FR, Hamilton H. Prediction of bone marrow iron findings for tests performed on peripherical blood. Am J Clin Pathol 1986; 85: 202-6.

2. Bennet RM, Holt PJL, Lewis SM. Role of reticuloendothelial system in the anemia of Rheumatoid Arthritis: A study using the 59Fe-labelled dextran model. Ann Rheum Dis 1974; 33: 147-52.

3. Weiss G. Iron and anemia of chronic disease. Kidney Intern 1999; 69: S12-7.

4. Cavill I, Bentley DP. Erythropoiesis in the anemia of Rheumatoid Arthritis. Br J Haematol 1982; 50: 582-90.

5. Means RT Jr. Advances in the anemia of chronic disease. Int J Hematol 1999; 70: 7-12.

6. Maury CP, Andersson LC, Teppo AM, Partanen S, Juvonen E. Mechanism of anaemia in Rheumatoid Arthritis: demonstration of raised interleukin-1beta concentrations in anaemic patients and of interleukin 1 mediated suppression of normal erythropoiesis and proliferation of human erythroleukaemia (HEL) cells in vitro. Ann Rheum Dis 1988; 47: 972-8.

7. Schooley JC, Kullgren B, Allison AC. Inhibition by interleukin-1 of the action of erythropoietin on erythroid precursors and its possible role in the pathogenesis of hypoplastic anaemias. Br J Haematol 1987; 67:11-7.

8. Voulgari PV, Kolios G, Papadopoulos GK, Katsaraki A, Seferiadiz $\mathrm{K}$, Drassos AA. Roled of cytokines in the pathogenesis of the anemia of chronic disease in rheumatoid arthritis. Clin Immunol 1999; 92: 153-160.

9. Prouse PJ, Harvey AR, Bonner B, Reid CD, Ansell BM, Gumpel M. Anaemia in juvenil arthritis: serum inhibition of normal erythropoiesis in vitro. Ann Rheum Dis 1987; 46: 127-34.

10. Dainiak N, Hardin J, Floyd V, Callahan M, Hoffman R. Humoral suppression of erythropoiesis in systemic lupus erythematosus (SLE) and rheumatoid arthritis. Am J Med 1980; 69: 537-44.

11. Dinarello CA. Interleukin-1 and the pathogenesis of the acute-phase response. N Eng J Med 1984; 311: 1413-8.

12. Dinarello CA, Clowes GH Jr, Gordon AH, Saravis CA, Wolff SM. Cleavage of human interleukin-1: isolation of a fragment from plasma of febrile humans and activated monocytes. J Immunol 1984; 133: 13328 .

13. Girardin E, Grau GE, Dayer JM, Roux-Lombard P, Lambert PH. Tumor necrosis factor and interleukin-1 in the serum of children with severe infectious purpura. N Eng J Med 1988; 319: 397-394.

14. The ACCP / SCCM Consensus Conferenece Commitee. Chest 1992; 20: $1644-55$.

15. Lee GR. The anemia of chronic disease. Semin Haematol 1983; 20: 6180 .

16. Wiitte DL, Kraener DF, Johnson GF. Prediction of bone marrow iron findings for test performed on peripherical blood. Am J Clin Pathol 1986; 85: 202-6.

17. Erslev AJ, Caro J, Miller O, Silver R. Renal and extrarrenal production in health and disease. Ann Clin Lab Sci 1980; 10: 250-7.

18. Birgegard G, Hellgren R, Caro J. Serum erytropoietin in rheumatoid arthritis and other inflammatory arthritides. Br J Haematol 1987; 65: 479-83.

19. Nielsen OJ, Andersen LS, Ludwigsen E, Bouchelouche P, Hansen TM. Birgens $\mathrm{H}$, et al. Anaemia of rheumatoid arthritis: serum erytropoietin concentrations and red cell distribution width in relation to iron status.
Ann Rheum Dis 1990; 49: 349-54.

20. Hochberg MC, Arnold CM, Hogans BB, Spivak JL. Serum immunoreactive erythropoietin in rheumatoid arthritis: impaired response to anemia. Arthritis Rheum 1988; 31: 1318-21.

21 Baer AN, Dessypris EN, Goldwasser E, Krantz SB. Blunted erythropoietin response to anemia in Rheumatoid Arthritis. Br J Haematol 1987; 66: 559-64.

22. Miller CB, Jones RJ, Piantadosi S, Abeloff MD, Spivak JL. Decreased erythropoietin response in patients with the anemia of cancer. $\mathrm{N}$ Eng $\mathbf{J}$ Med 1990; 322: 1689-92.

23. Spivack JL, Barnes DC, Fuchs E, Quinn TC. Serum immunoreactive erythropoietin in HIV-infected patients. JAMA 1989; 3104-7.

24. Ebrahim O, Folb PI, Robson SC, Jacobs P. Blunted erythropoietin response to anemia in tuberculosis. Eur J Haematol 1995; 55: 251-4.

25. Camacho J, Arnalich F, Zamorano AF, Vázquez JJ. Serum erythropoietin levels in the anaemia of chronic disorders. J Intern Med 1991; 229: 49-54.

26. Camacho J. Poveda F. Zamorano AF. Valencia ME. Vazquez JJ. Arnalich F. Serum erythropoietin levels in anaemic patients with advanced human immunodeficiency infection.Br J Haematol 1992; 82: 608-14.

27. Pincus T, Olsen NJ, Russell IJ, Wolfe F, Harris ER, Schnitzer TJ, et al. Multicenter study of recombinant human erythropoietin in correction of anemia in rheumatoid arthritis. Am J Med 1990; 89: 161-8.

28. Salvarini C, Lasagni D, Casali B, et al. Recombinant human erythropoietin therapy in patients with rheumatoid arthritis with the anemia of chronic disease. J Rheumatol 1991; 181: 1168-71.

29. Tracey KJ, Wei H, Manogue KR, Fong Y, Hesse DG, Nguyen HT, et al. Cachectin / tumor necrosis induces cachesia, anemia and inflammation. J Exp Med 1988; 167: 1211-27.

30. Blick M, Sherwin SA. Rosenblum M, Gutterman J. Phase I study of recombinant tumor necrosis factor in cancer patients. Cancer Res 1987; 47: 2986-9.

31. Greendyke RM, Sharma K, Gifford FR. Serum erythropoietin and selected other cytokines in patinets with anemia of chronic disease. Am J Clin Pathol 1994; 101: 338-41.

32. Roodman GD, Bird A, Hutzler D. Montgomery W. Tumor necrosis factor alpha and hematopoietic progenitors: Effects of tumor necrosis factor on the growth of erythroid progenitors CFU-E and BFU-E and the hematopoietic cell lines K562, HL60, and HEL cells. Exp Hematol 1987; 15: 928-35.

33. Akahane K. Hosoi T. Urabe A. Kawakami M. Takaku F. Effects of recombinant human tumor necrosis factor (rhTNF) on normal human and mouse hemopoietic progenitors cells. Int J Cell Cloning 1987; 5: 16-26.

34. Means RT, Krantz SB. Progress in understanding the pathogenesis of the anemia of chronic disease. Blood 1992; 80: 1639-47.

35. Means RT, Krantz SB. Progress in understanding the pathogenesis of the anemia of chronic disesase. Blood 1992; 80: 1639-47.

36. Asano S, Okano A, Ozawa K, Nakahata T, Ishibashi T, Koike K, et al In vivo effects of recombinant human interleukin-6 primates: Stimulated production of platelets. Blood 1990; 75: 1602.

37. Vreugdenhil G, Lowenberg B, van Eijk HG, Swaak AJ. Anaemia of chronic disease in rheumatoid arthritis: Raised serum interleukin-6 levels and the effects of IL-6 and anti-IL-6 on in vitro erythropoiesis. Rheumatol Int 1990; 10: 127-30. 